\title{
Urgences
}

\section{L'effet prismatique}

\section{Bernard Pozier}

Numéro 26, décembre 1989

Des textes qui chantent

URI : https://id.erudit.org/iderudit/025559ar

DOI : https://doi.org/10.7202/025559ar

Aller au sommaire du numéro

Éditeur(s)

Urgences

ISSN

0226-9554 (imprimé)

1927-3924 (numérique)

Découvrir la revue

Citer cet article

Pozier, B. (1989). L'effet prismatique. Urgences, (26), 93-99.

https://doi.org/10.7202/025559ar

Ce document est protégé par la loi sur le droit d'auteur. L'utilisation des services d'Érudit (y compris la reproduction) est assujettie à sa politique d'utilisation que vous pouvez consulter en ligne.

https://apropos.erudit.org/fr/usagers/politique-dutilisation/
Cet article est diffusé et préservé par Érudit.

Érudit est un consortium interuniversitaire sans but lucratif composé de l’Université de Montréal, l'Université Laval et l'Université du Québec à Montréal. Il a pour mission la promotion et la valorisation de la recherche. https://www.erudit.org/fr/ 


\section{L'effet prismatique Bernard Pozier}

On achève blen les rockers (It's only rock n' roll...) $)^{1}$

On a tous envie de partir Quand rien ne va qui vaille On voudrait tout oublier Quand on n'a rien à perdre

5 Dans des hôtels de $3^{\theta}$ ordre Où trainent les fleurs du mal La détresse nous attend Quand plus personne ne vient

On a tous l'idée de s'enfuir

10 Par la porte d'en arrière Mais on finit par rester

Sur place devant la glace Qui déforme tout

On achève bien les rockers

15 Lorsqu'ils ont les bleus au cœur Et lorsqu'ils ont les idées larges S'entêtent à vivre leur vie en marge

Quand on est seul dans sa chambre La nuit vient nous chercher

20 Comme un vieux souvenir Du bout de la mémoire $Y$ ' a toujours un con qui revient Chercher son perfecto Quand arrive le matin

25 On se prend pour Rimbaud It's only rock n' roll but i like it...

On achève bien les rockers Lorsqu'ils qu'ils ont les bleus au cœur Ils sont comme des gitans blessés 30 Au fond des cafés oubliés 
94

On achève bien les rockers

Lorsqu'ils ont les bleus au coeur

Ils portent des blousons usés

Et des blue-jeans déchirés

On a tous l'idée de partir

Quand rien ne va qui vaille

On voudrait tout oublier

Quand on n'a rien à perdre

On a tous l'idée de s'enfuir

40 Par la porte d'en arrière

Mais on finit par rester

Carrément rocker

Totalement rocker

Strictement rocker

45 Essentiellement rocker

On achève bien les rockers

Lorsqu'ils ont les bleus au coeur

Et lorsqu'ils ont les idées larges

S'entêtent à vivre leur vie en marge

50 On achève bien les rockers

Lorsqu'ils ont les bleus au cœur

Ils sont comme des chevaux sauvages

Au fond des bars sans éclairage

On achève bien les rockers

55 Lorsqu'ils ont les bleus au coeur

Ils sont comme des gitans blessés

Au fond des cafés oubliés

On achève bien les rockers

Lorsqu'ils ont les bleus au cœur

60 Ils sont comme des chevaux sauvages

Au fond des bars sans éclairage

Carrément rocker

Totalement rocker

Absolument rocker

Lucien Francœur est un grand frondeur mythomane et lorsqu'il lit ou entend quelqu'un qu'il aime, il a le goût de se prendre pour ses idoles. Cependant, au lieu de se limiter à faire ses imitations dans sa tête, sur ses feuilles ou dans son miroir, comme chacun, il ose s'aventurer à se prendre pour une star sur les scènes publiques de la chanson et de la littérature. 
Ce qui fait le charme de l'entreprise discographique du poète Lucien Francœur, c'est bien sûr un certain humour, de même que le mixage des langages. L'ironie, la parodie et la caricature sont fréquemment esquissées aux lignes ou aux notes de la majorité des chansons/monologues qui sont toujours à peu près, en fait, des "raps", façon Lucien. Le métissage linguistique, quant à lui, s'opère au croisement des vocabulaires parisien, américain et typiquement québécois.

Cependant, le principal attrait de toute la discographie du poète réside sans doute dans la stratégie déployée dans l'écriture, comme dans la texture musicale, dans cette façon bien personnelle de tricoter les matériaux culturels: la mythologie rock n' roll, la culture populaire, l'histoire littéraire, les notes de voyage, l'actualité...

Toutes ces données, emmagasinées dans le Macintosh de Francœur, s'emmêlent et resurgissent sous la forme de petites histoires ou comme un jeu vidéo où les mots et les phrases seraient des trappes ou des attrapes dans un panorama de clins d'œil. En lisant ou en écoutant Lucien Francœur, on se rend vite compte que ses mots sont des feintes et qu'ils rebondissent illico dans notre cerveau pour prendre d'autres routes, animer d'autres réseaux signifiants.

Les textes, dans leur apparente simplicité, camouflent de l'indirect et nous renvoient toujours ailleurs, comme si le parolier ne disait jamais vraiment les mots qu'il prononce et comme si tout cela n'était signe que d'autres choses, de lignes de fuite à emprunter dans tous les sens. Voilà ce que j'appellerais, chez Lucien Francœur, l'effet prismatique, cette tactique qui cause la diffraction de notre pensée.

Cette même stratégie textuelle se déploie évidemment dans son hymne rock n' roll intitulé On achève bien les rockers. Suivons à la trace le toboggan des mots...

Dès le titre, on s'enfarge dans la référence, la phrase d'envoi calquée sur un film célébré, On achève bien les chevaux (il en galopera d'ailleurs un peu plus loin, mais il n'y aura pas de marathon de danse). Le sous-titre, lui, laisse le calque pour la pure citation, une vieille moto rock n' roll.

Un peu plus bas, on peut lire "les fleurs du mal" en se rappelant évidemment Baudelaire, mais aussi la chanson de Bernard Lavilliers, La fleur du mal, parue sur l'album Tout est permis rien n'est possible. Et puis le personnage de la 
96

chanson, envahi par le «spleen» de fin de siècle, se regarde dans la "glace", celle de lhiver québécois ou celle du miroir de la chanson L'espion sur l'album Francour: Le retour de Johnny Frisson. Ce même album fournit une autre ligne clé, celle des "bleus au cœur», déjà présente dans la pièce Cheap licks (pour Françoise Hardy), à qui « le Larsen » faisait * des bleus au cœur *.

De cette manière, Francœur fait un clin d'œil à ses fans, comme on se fait des signes entre initiés; on peut aussi voir cela comme une forme d'autopublicité. Ce phénomène, fréquent dans l'entreprise de l'auteur, permet des aller-retour en tous sens entre le corpus littéraire et celui de la chanson.

À ce titre, il y a dans On achève bien les rockers une strophe particulièrement éloquente, la sixième, qui est aussi la plus française. Ironiquement, celle-ci commence par la seule prononciation québécoise ( $\left(\mathrm{Y}^{\prime}\right.$ a»), d'ailleurs devenue le fétiche de Michel Barrette, un copain de Lucien, avec qui il s'est fait prendre à l'émission du " farceur * Marcel Béliveau.

Dans ce passage, trois mots frappent, par leur écho tout à fait parisien: "con ", "perfecto * et "Rimbaud». Les deux derniers apparaissent particulièrement efficaces sur le plan des références qu'ils éveillent. Ce "perfecto» rappelle ou publicise le recueil Perfecto nuit (VLB, 1988), tandis que * Rimbaud * semble utilisé par Francœur pour prendre en charge à la fois la chanson/émission/collection Café Rimbaud, la chanson Une saison en enfer, autre séquence de l'époque Aut' chose, et, plus encore, le recueil Si Rimbaud pouvait me lire (Noroit, 1987), car celui-ci s'exerce à appliquer les mots de la chanson On se prend pour Rimbaud. Cette prétention-là était aussi déjà signalée dans Une prière rock (L'hexagone, 1985) par le jeu de la mise en scène (exergue et quatrième de couverture) et très explicitement dans plusieurs autres textes.

L'emploi du mot gitan rejoint également une des images obsessives de l'écriture du Francœur des quelques dernières années. Il nous ramène d'abord au titre même de l'album où est gravé On achève bien les rockers qui est: Les gitans reviennent toujours; celui-ci comprend d'ailleurs en plus une chanson qui s'intitule L'amour en Gitanie, tout comme il y avait un Gypsie rock sur Dernière vision. Et il ne faut pas non plus négliger de penser aux recueils Des images pour une gitane (Orphée, 1981), La petite bohémia des arcanes (Gitanéria, 1985) et L'enchanteur et la bohémienne endormie (Gitanéria, 1985), tous parus à petits tirages: un clin d'œil pour spécialistes! 
Sont également présents les «blousons * et *blue-jeans *, comme des fétiches rock n' roll, comme un écho de Blue jeans sur la plage, parue sur l'album Une nuit comme une autre. Quant à ces rockers dont il est question tout au long de la chanson (mot que Lucien s'entête ici à orthographier à l'américaine plutôt qu'à la française: "rockers" plutôt que «rockeurs»), il nous faut les rapprocher de ceux qu'il appelait Les rockeurs sanctifiés dans son livre calligraphié, paru à l'Hexagone et gagnant du prix Émile-Nelligan.

En effet, les nombreux adverbes et leur gradation nous obligent à y lire un sens religieux: on est au rock comme on est chevalier ou comme on devient saint, car dans l'imagerie de Francœur on l'est " carrément ", " totalement ", " strictement * et finalement * essentiellement *, dans une démarche nécessairement globale et absolutiste. À la dernière ligne apparaît d'ailleurs l'adverbe «absolument», à la fois employé dans le sens de "résolument " et de « d'une manière absolue ".

Du chevalier au cheval, il n'y a qu'un pas et ces animauxlà, le titre les avait annoncés, donc voici que ses héros * sont comme des chevaux sauvages $»$ : ceux que l'on entend sur le Sticky fingers des Rolling Stones ou ceux, plus proches dans le temps, du hit de Gino Vanelli? Et les besoins de la rime nous mènent d'un enclos à un autre de l'ère hippique (est-ce que cela viendrait de "hippie $»$ ?) à l'espace où se cassent bien des galops de l'imagination, le «bar», dépeint par: «au fond des bars sans éclairage " (v. 53), qu'il convient de mettre en parallèle, non seulement avec le Café Rimbaud, mais aussi avec un autre titre de l'album, celui de la pièce précédente: Le bar d' la dernière chance. Et bien sûr, de *dernière chance* à * on achève», l'enchaînement ne saurait être plus logique.

Voilà un peu où les mots nous entraînent, comment ils nous font nomades, comment ils se déterritorialisent, partant du texte de la chanson pour se reterritorialiser dans nos ailleurs culturels personnels et collectifs, dans un mouvement tout deleuzien dont se réclamerait évidemment Lucien. C'est en ce sens que les textes de Lucien Francœur sont, quelque part, exactement l'inverse d'un texte de chanson, en ce qu'ils ne sont pas immédiatement saisissables par tous, du moins pas dans toutes leurs dimensions, à cause du jeu des allusions qui en font une écriture surcodée, qui exigent une lecture "culturelle", c'est-à-dire une connaissance au moins superficielle des corpus littéraire et rock n'roll, ce qui n'est pas 
98

nécessairement à la portée de n'importe quel auditeur de radio, atteint par hasard.

Côté musique et sonorisation, il y a du Gerry Boulet làdessous, dans les voix et dans les claviers, dans le travail de simplification du texte qu'il a exigé de Francœur, dans le traitement aussi de la voix de l'interprète à qui il amène du renfort. La puissance musicale est assurée par l'ajout de cuivres dont Francœur avait rarement fait usage auparavant. Et il y a ce chœur auquel tenait absolument Francœur pour faire un peu son We are the world ou son petit Band-aid personnel.

Notons particulièrement les présences, dont certaines surprenantes, de Jacques Blais (guitariste), Sylvie Boucher, Jean Dorais (autre «guitar hero * québécois), Marc (Comme j'ai toujours envie d'aimer) Hamilton, Pierre Létourneau ou des plus jeunes: Tess et Mario Trudel. Enfin, sur le plan du mixage, un passage vers la fin intercale les voix, un peu comme on l'avait fait sur An american prayer de Jim Morrison. Et quand on connaît l'attachement de Francœur pour celui-là, faut croire que ce n'est pas tout à fait un hasard.

Sur le plan thématique, l'un des éléments notoires de cette thêatralisation rock n' rollienne, c'est certes le thème du nomadisme avorté, comme si Kerouac n'avait jamais pu quitter Mémère pour le moindre voyage. $Y$ aurait-il là-dedans une forme d'impuissance à exister, foncièrement, viscéralement et vicieusement québécoise? En effet, si * On a tous envie de partir " (v. 1), si «On a tous l'idée de s'enfuir» (v. 9), il n'en demeure pas moins que l'« on finit par rester / Sur place devant la glace / Qui déforme tout * (v. 11-13). Et la seule image qui sauve est celle de se dire marginal quand, au *il * de la description, s'emmêlent le *on* et le "nous" pour accentuer notre identification.

Cette superposition du * on *, techniquement impersonnel, au * nous *, exactement pluriel, tout à fait québécoise, pourrait justement dénoter une absence d'identification réelle. Ici, plus spécifiquement, elle vise aussi discrètement, un peu comme un paradoxe rusé, à ce que chacun se sente impliqué par ce que dit la chanson, que chacun se prenne pour un membre de cette marginalité décrite et célébrée.

Ce On achève bien les rockers, c'est aussi à la fois la glorification d'une frange parasociale et, en sourdine, la répétition d'une vieille marotte de Francœur: «Le rock se meurt au Québec. » 
Sur le plan de la structure, la construction s'échafaude sur douze strophes de vers relativement brefs où les rimes sont fréquentes, mais pas constantes. Les strophes aussi sont irrégulières: si la moitié des strophes ont quatre vers chacune, on en trouve aussi une de seize vers et une d'un seul vers, en anglais de surcroît. Les énoncés évoluent donc ici en pleine liberté; d'ailleurs, il n'y a pas, à proprement parler, de refrains ni de couplets. Plusieurs lignes sont répétées, mais dans des agencements variés; seule la neuvième strophe reprend intégralement la première, sans y ajouter de variantes, sans l'entrecroiser avec d'autres passages. Voilà une tactique de boutures et de rhizomes bien deleuzienne.

Francœur, depuis toujours, prêche en effet le nomadisme; chez lui, donc, tout se déplace. Il a la bougeotte et demeure toujours en transit: entre poésie et chanson, entre Paris, Montréal et Los Angeles, mais aussi dans son interprétation même. Ici, il hésite entre dire et chanter, comme il oscille entre les mythologies et les vocabulaires français, américain et québécois, comme aussi il alterne constamment dans ses propos entre la mort et la renaissance du rock et de la poésie.

De facture simple, la chanson On achève bien les rockers de Lucien Francour n'en demande pas moins une lecture complexe et plurielle, à travers plusieurs épaisseurs de signification, les mots dévoilant plusieurs facettes, selon l'angle de la lecture ou, plus précisément, selon celui du souvenir du lecteur et/ou de l'auditeur.

Des reflets de toutes sortes de choses nous entrent par les oreilles et se promènent dans toutes sortes de recoins de nos cerveaux où joue le poète qui se prend pour un chanteur, à moins que ce soit le rockeur qui se prenne pour un poète. En tout cas, les mots circulent.

once i had a little game

i liked to crawl back into my brain

$i$ think you know the game i mean

\section{Jim Morrison \\ Celebration of the lizard}

"I think you know the game I mean ", au fond toute la stratégie d'écriture de Francœur est là, tout s'inscrit aux signes de la complicité... « now you should try this little game *... disait Morrison à Lucien, «essayez ce petit jeu * redit Lucien à qui l'écoute. 